\title{
The Blazhko RR Lyrae variables and phase modulation in binary systems
}

\author{
Hiromoto Shibahashi ${ }^{1, \star}$ \\ ${ }^{1}$ University of Tokyo, Department of Astronomy, Tokyo 113-0033, Tokyo, Japan
}

\begin{abstract}
With the help of the Fourier transform, I verify that some stars classified as Blazhko variables are actually regularly pulsating RR Lyrae stars in binary systems.
\end{abstract}

\section{Introduction and summary}

The Blazhko phenomenon is the periodic variation in pulsation amplitude or phase (or equivalently, frequency) seen in a large fraction of RR Lyrae stars ([1,2]), and it still remains a long-lived enigma to be explained. However, phase modulation is also seen in pulsating stars in binary systems, due to the light-travel time effect $([3,4])$. Some stars classified as Blazhko variables might be then explained as binary systems. We should carefully discriminate between intrinsic phase modulation in RR Lyrae stars and phase modulation due to binarity. Hajdu et al. ([5]) adopted the O-C method to detect binarity. Another way to discriminate these two causes more clearly is the Fourier transform $([6,7])$. By carefully investigating the multiplets seen in the Fourier spectra of RR Lyrae stars, I verify that some of the stars classified as Blazhko variables are actually non-Blazhko RR Lyrae stars in a binary system, and conversely show that some stars classified as binary systems are actually not binaries.

\section{The light-travel time effect and evidence for binarity}

Let us consider a star pulsating with a frequency $v_{0}$ and its harmonics in a binary. The observed luminosity variation at time $t$ is then given by

$$
\Delta L(t)=\sum_{k} A_{k} \cos 2 \pi\left\{k v_{0}\left[t-\frac{1}{c} \int_{0}^{t} v_{\mathrm{rad}}\left(t^{\prime}\right) d t^{\prime}\right]+\phi_{k}\right\},
$$

where $c$ is the speed of light and $v_{\text {rad }}(t)$ denotes the radial velocity, due to the orbital motion, of the pulsating star at the time $t^{\prime}$, where the epoch is the time at which the star passes the nodal point directed towards us.

The Fourier transform of eq. (1) leads to ([7]) (i) multiplets with an equal spacing in frequency, equal to the orbital frequency, (ii) the amplitude ratio between the first sidelobe and the central component of each multiplet being proportional to the oscillation frequency, $k v_{0}$, and (iii) the phase difference between the central component and the first sidelobes of each multiplet being $\pi / 2$. These features discriminate the regularly pulsating RR Lyrae stars in a binary system from the Blazhko RR Lyrae variables.

\footnotetext{
${ }^{\star}$ shibahashi@astron.s.u-tokyo.ac.jp
} 


\section{Stars showing features of binarity}

A good example is OGLE-BLG-RRLYR-06498. Figure 1(a) shows the amplitude spectrum around the highest peak, $v_{0}$. The conspicious side peaks are caused by a year-gap of observations appearing in the window spectrum. After pre-whitening $v_{0}$, we see clearly a pair of sidelobes of a triplet around $v_{0}$ (Fig. 1(b)). As seen in Figure 1(c), the frequency spacings between members of this and other multiplets are equal. The amplitude ratio between the sidelobe and the central component of each triplet, shown in Figure 1(d), is proportional to frequency, being consistent with binarity. Figure 1(e) shows that the phase difference between the mean of the sidelobes and the central component of triplets is $\pi / 2$, again consistent with binarity. Hence, we conclude that the phase modulation seen in this star is caused by orbital motion rather than an intrinsic Blazhko effect.
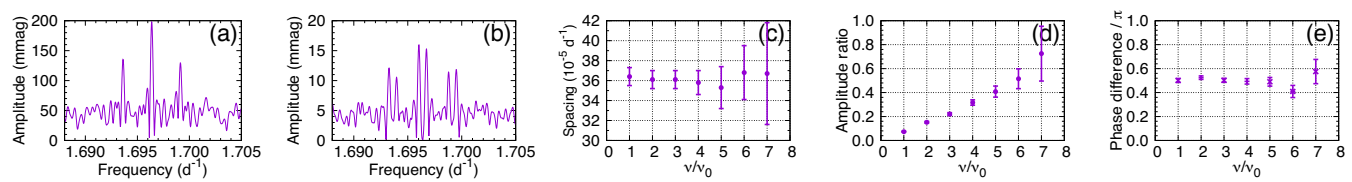

Figure 1. The amplitude spectrum of OGLE-BLG-RRLYR-06498 and its features, showing binarity.

\section{Stars showing features of the Blazhko cycle}

An example is KIC 10789273. Figure 2(a) shows the amplitude spectrum around the highest peak, $v_{0}$. Pre-whitening of $v_{0}$ makes a triplet visible as shown in Figure 2(b). Figure 2(c) shows that the frequency spacings among multiplets are equal. The amplitude ratio between the sidelobe and the central component of triplets, shown in Figure 2(d), is, however, not proportional to frequency, being inconsistent with binarity. Figure 2(e) shows that the phase difference between the mean of the sidelobes and the central component of triplets is not $\pi / 2$ at all, being inconsistent with binarity.
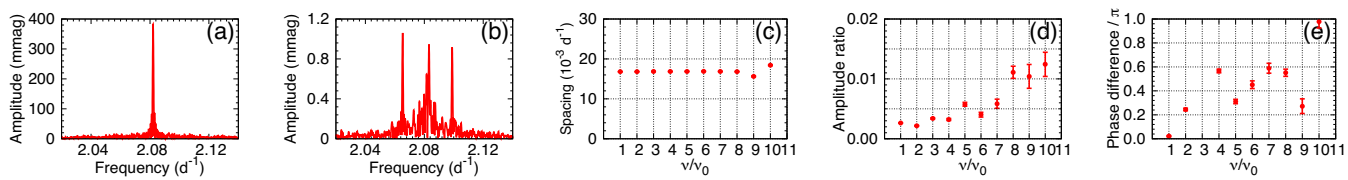

Figure 2. The amplitude spectrum of KIC 10789273 and its features are inconsistent with binarity.

\section{References}

[1] Blažko, S., AN, 175, 325 (1907)

[2] Shapley, H., ApJ, 43, 217 (1916)

[3] Murphy, S. J., Bedding, T. R., Shibahashi, H., Kurtz, D. W., \& Kjeldsen, H., MNRAS, 441, 2515 (2014)

[4] Murphy, S. J., \& Shibahashi, H., MNRAS, 450, 4475 (2015)

[5] Hajdu, G., Catelan, M., Jurcsik, J., Dékány, I., Drake, A. J., \& Marquette, J.-B., MNRAS, 449, L113 (2015)

[6] Shibahashi, H., \& Kurtz, D. W., MNRAS, 422, 738 (2012)

[7] Shibahashi, H., Kurtz, D. W., \& Murphy, S. J., MNRAS, 450, 3999 (2015) 